\title{
BMJ Open Screening of gestational diabetes mellitus in early pregnancy by oral glucose tolerance test and glycosylated fibronectin: study protocol for an international, prospective, multicentre cohort trial
}

\author{
E A Huhn, ${ }^{1}$ T Fischer, ${ }^{2}$ C S Göbl, ${ }^{3}$ M Todesco Bernasconi, ${ }^{4} \mathrm{M}$ Kreft, ${ }^{5} \mathrm{M}$ Kunze, ${ }^{6}$ \\ A Schoetzau, ${ }^{1}$ E DölzImüller, ${ }^{2}$ W Eppel, ${ }^{3} \mathrm{P}$ Husslein, ${ }^{3} \mathrm{~N}$ Ochsenbein-Koelble, ${ }^{5}$ \\ R Zimmermann, ${ }^{5}$ E Bäz, ${ }^{6}$ H Prömpeler, ${ }^{6}$ E Bruder, ${ }^{7}$ S Hahn, ${ }^{8}$ I Hoesli ${ }^{1}$
}

To cite: Huhn EA, Fischer T, Göbl CS, et al. Screening of gestational diabetes mellitus in early pregnancy by oral glucose tolerance test and glycosylated fibronectin: study protocol for an international, prospective, multicentre cohort trial. BMJ Open 2016;6:e012115 doi:10.1136/bmjopen-2016012115

- Prepublication history and additional material is available. To view please visit the journal (http://dx.doi.org/ 10.1136/bmjopen-2016012115).

Received 7 April 2016 Revised 13 July 2016 Accepted 24 August 2016

CrossMark

For numbered affiliations see end of article.

Correspondence to

Dr EA Huhn;

evelyn.huhn@usb.ch

\section{ABSTRACT}

Introduction: As the accurate diagnosis and treatment of gestational diabetes mellitus (GDM) is of increasing importance; new diagnostic approaches for the assessment of GDM in early pregnancy were recently suggested. We evaluate the diagnostic power of an 'early' oral glucose tolerance test (OGTT) $75 \mathrm{~g}$ and glycosylated fibronectin (glyFn) for GDM screening in a normal cohort.

Methods and analysis: In a prospective cohort study, 748 singleton pregnancies are recruited in 6 centres in Switzerland, Austria and Germany. Women are screened for pre-existing diabetes mellitus and GDM by an 'early' OGTT $75 \mathrm{~g}$ and/or the new biomarker, glyFn, at 12-15 weeks of gestation. Different screening strategies are compared to evaluate the impact on detection of GDM by an OGTT $75 \mathrm{~g}$ at 24-28 weeks of gestation as recommended by the International Association of Diabetes and Pregnancy Study Groups (IADPSG). A new screening algorithm is created by using multivariable risk estimation based on 'early' OGTT $75 \mathrm{~g}$ and/or glyFn results, incorporating maternal risk factors. Recruitment began in May 2014.

Ethics and dissemination: This study received ethical approval from the ethics committees in Basel, Zurich, Vienna, Salzburg and Freiburg. It was registered under http://www.ClinicalTrials.gov (NCT02035059) on 12 January 2014. Data will be presented at international conferences and published in peer-reviewed journals.

Trial registration number: NCT02035059.

\section{INTRODUCTION}

Gestational diabetes mellitus (GDM) is defined as diabetes diagnosed during pregnancy that is not clearly overt diabetes. ${ }^{1}$ The increasing number of women with

\section{Strengths and limitations of this study}

- This is an international, prospective, multicentre cohort trial recruiting at six centres in Switzerland, Austria and Germany.

- It is the first study to assess an 'early' oral glucose tolerance test $75 \mathrm{~g}$ and novel biomarkers like glycosylated fibronectin for screening of gestational diabetes mellitus in early pregnancy.

- The recruitment of 748 pregnant women is planned. We have designed the study to be sufficiently powered to compare the different early screening approaches with the detection of gestational diabetes mellitus at 24-28 weeks of gestation.

- This study may be underpowered for the evaluation of neonatal outcomes like large for gestational age infants, neonatal hypoglycaemia, shoulder dystocia or birth trauma (secondary outcomes).

undiagnosed type 2 diabetes mellitus (T2DM) in pregnancy has led to the recommendation of screening women with risk factors for preexisting diabetes at the first antenatal visit. GDM is still diagnosed in the late second or early third trimester, because accurate diagnostic approaches for GDM assessment in first trimester are still lacking. ${ }^{2}$

GDM is associated with adverse maternal and perinatal outcomes, such as fetal overgrowth, shoulder dystocia, operative delivery, birth injury, pre-eclampsia, haemorrhage and preterm delivery, ${ }^{3-5}$ but also a sevenfold higher risk of the mother developing T2DM after pregnancy. ${ }^{6}$ In addition, the maternal metabolic milieu was also identified as a key 
determinant for the susceptibility to obesity, metabolic syndrome and T2DM in the offspring, ${ }^{7}$ a phenomenon often described as 'fetal programming'.

The current-but still widely discussed-standard of care in GDM screening is the oral glucose tolerance test (OGTT) of $75 \mathrm{~g}$ glucose performed late at 24-28 weeks of gestation as recommended by the International Association of Diabetes and Pregnancy Study Groups (IADPSG). ${ }^{8}$ The new screening thresholds are based on the results of a large prospective cohort multicentre trial, the Hyperglycaemia and Adverse Pregnancy Outcome (HAPO) study. ${ }^{4}$ The aim of the HAPO study was to associate the degree of maternal glycaemia with adverse perinatal outcome, such as large for gestational age (LGA) infants, neonatal hypoglycaemia and caesarean section rates. The results showed no obvious threshold, but rather a continuous increase of these adverse outcomes across the range of glucose concentrations. The IADPSG criteria resulted in a considerable increase in GDM prevalence of $17.8 \%$, a detection rate of $83 \%$ for adverse outcome and a positive predictive value of $16 \%{ }^{8}$

An early and rapid diagnosis of GDM even before 24 weeks of gestation is desirable. By targeted early intervention including physical activity, moderate diet or insulin/drug therapy starting in the first trimester, rates of macrosomia (birth weight $>4000 \mathrm{~g}$ ) or LGA (LGA=birth weight $>90$ th centile) infants, operative vaginal delivery and perinatal morbidity could be possibly reduced. Moreover, there could be a long-term downstream effect on the offspring, thereby leading to considerable savings in healthcare costs by possibly decreased prevalence of generational transmission of metabolic diseases. But further research is necessary to evaluate the effects of an early intervention on shortterm and long-term outcomes for mother and child.

We propose that an 'early' OGTT combined with maternal history, maternal condition and promising new biomarkers such as glycosylated fibronectin (glyFn) could diagnose similarly GDM, even in the first trimester. Rasanen et al published a study in September 2013 introducing glyFn as a new early GDM screening approach with an area under the curve (AUC) of 0.91 and $95 \%$ CI of 0.87 to 0.96 , a positive predictive value of $63 \%$ and a negative predictive value of $95 \%$. Although some predictors of GDM have been studied retrospectively, no study to date has considered the use of promising new biomarkers combined with an 'early' OGTT and maternal risk factors evaluation in first trimester of pregnancy.

\section{STUDY OBJECTIVES}

\section{Primary objective}

The use of the 'early' OGTT $75 \mathrm{~g}$ and/or the new biomarker, glyFn, as a new screening approach in late first/ early second trimester will be evaluated and compared with GDM diagnosis by OGTT $75 \mathrm{~g}$ at 24-28 weeks of gestation.

\section{Secondary objectives}

1. A new screening algorithm will be created by using multivariable risk estimation based on 'early' OGTT $75 \mathrm{~g}$ and/or glyFn results, incorporating maternal risk factors.

2. The significance of the association between glyFn, 'early' OGTT $75 \mathrm{~g}$ and maternal body mass index and/or clinical conditions including chronic hypertension, pregnancy-induced hypertension or preeclampsia and fetal conditions such as intrauterine growth restriction will be evaluated.

\section{METHODS}

This study protocol was developed on the basis of Standard Protocol Items: Recommendations for Interventional Trials (SPIRIT; see online supplementary 'SPIRIT checklist' for further details).

\section{Study settings/design}

This is an international, prospective, multicentre cohort trial conducted at one secondary and five tertiary referral centres in Switzerland, Austria and Germany. Study recruitment started primarily at the coordination centre at University Hospital Basel on 1 May 2014. All other centres started recruitment consecutively until the end of March 2016. Recruitment is expected to last until December 2017 (see figure 1 for details about the expected time frame). The aim is to enrol 748 women at 12-15 weeks of gestation with a minimum recruitment of 50 women planned for each centre depending on size and time of recruitment (Aarau: $n=50$, Basel: $n=358$, Freiburg: n=60, Salzburg: $n=100$, Vienna: $n=100$, Zurich: $\mathrm{n}=80$ ). Fifty per cent of eligible women are expected to accept participation. The trial was registered under http://www.ClinicalTrials.gov under NCT02035059 on 12 January 2014.

\section{Recruitment and informed consent}

Participants are identified at their first antenatal visit between 6 and 15 weeks of gestation. The investigator or obstetrician in charge informs the women about all aspects pertaining to the trial. The informed consent includes permission for gathering data from medical records and the optional storage of blood for a maximum of 10 years for additional analyses related to the current study. Participants are informed that trial participation is voluntary and that they are free to withdraw without any effects on subsequent care. All members of the research team are aware of the guidelines for good clinical practice for obtaining consent. ${ }^{9}$

\section{Eligibility criteria}

Inclusion criteria are

- Women at least 18 years of age and not under guardianship;

- Healthy singleton pregnancy after spontaneous conception or after fertility treatment; 


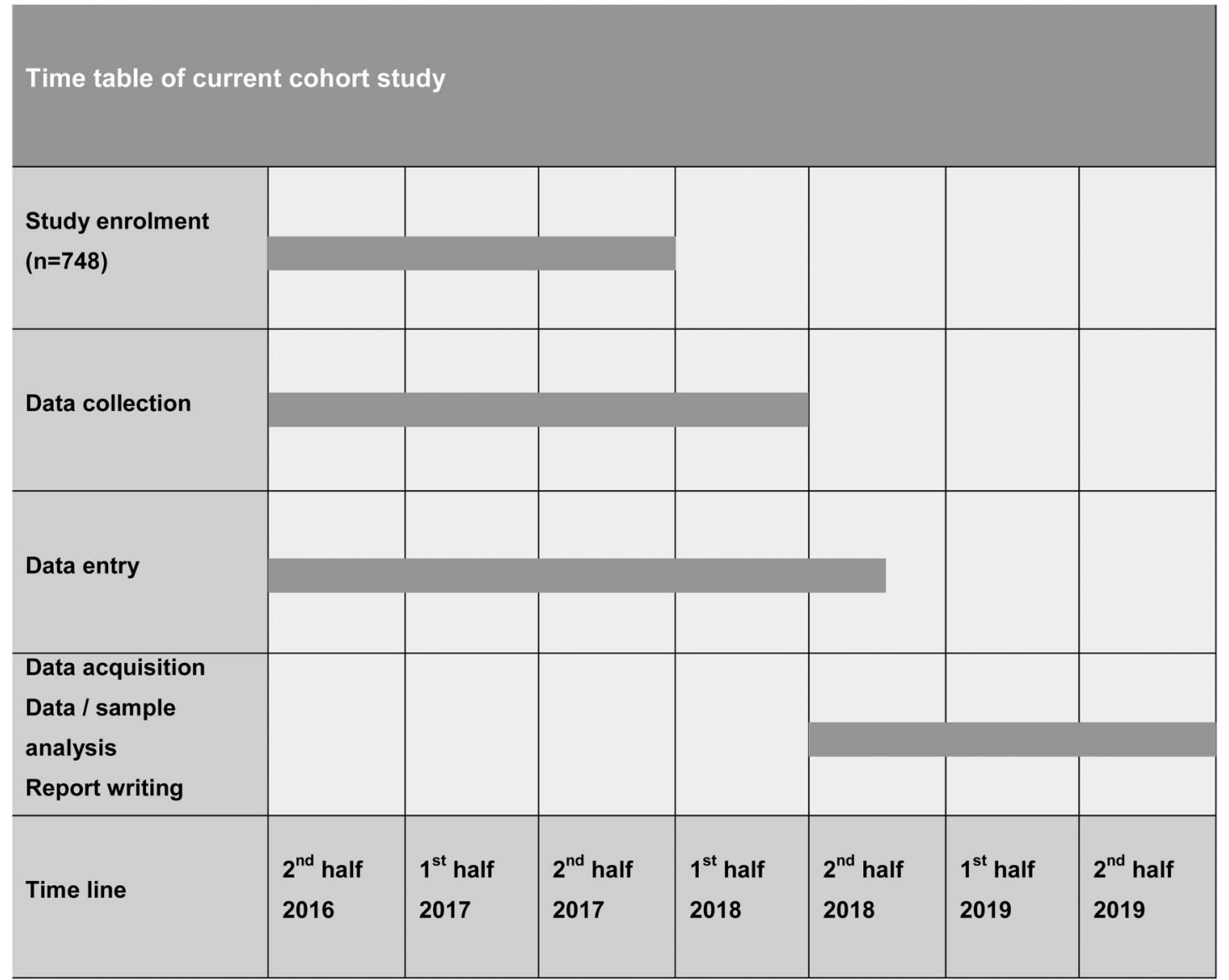

Figure 1 Expected time frame.

- Six to 15 weeks of gestation;

- Signed informed consent.

\section{Exclusion criteria are}

- Previous bariatric surgery;

- Known pre-existing diabetes mellitus or under treatment with metformin;

- Known chronic infection like hepatitis or HIV or chronic kidney, liver or heart disease;

- Known maternal history of hypertensive diseases in a previous pregnancy and now under prophylactic acetylsalicylate treatment;

- Fetal genetic, chromosomal or intervention-requiring morphological abnormalities;

- The inability to read and/or understand the participant's information sheet.

\section{Study procedure}

All healthy pregnant patients with regular care at the participating hospitals are counselled and asked at 6-15 weeks of gestation to participate. At $10+0$ to $13+6$ weeks of gestation, all women have to undergo a first trimester ultrasound scan which is standard care at participating sites. The ultrasound scan is used to confirm gestational age, diagnose any major fetal abnormalities and optionally measure fetal nuchal translucency thickness, which together with maternal free $\beta$-chorionic gonadotropin and pregnancy-associated plasma protein $\mathrm{A}$, is used for screening for chromosomal abnormalities. In addition, if informed consent had been given during the first antenatal visit or at the time of the first trimester scan, the maternal history and condition are assessed, and blood for biomarker analysis and for the 'early' OGTT $75 \mathrm{~g}$ is drawn at the study visit at $12-15$ weeks of gestation. The 'early' OGTT $75 \mathrm{~g}$ is compared with plasma glucose results obtained at 24-28 weeks of gestation after the OGTT $75 \mathrm{~g}$. No additional visit is necessary besides the standard routine antenatal care visits.

\section{GlyFn and 'early' OGTT $75 \mathrm{~g}$}

All participants are instructed to fast for at least 10 hours before sampling. Two fasting glucose samples are taken. One sample is collected for storage of two aliquots $(2 \times 1 \mathrm{~mL})$ at $-80^{\circ} \mathrm{C}$ for later analysis of glyFn and another sample for the fasting plasma glucose (FPG) value. After intake of the $75 \mathrm{~g}$ glucose load, blood samples are drawn 60 and 120 min later for the determination of glucose levels. Plasma glucose is measured by an automated colorimetric-enzymatic method (hexokinase/ glucose-6-phosphate-dehydrogenase) on a Hitachi/RocheModular P analyser. GlyFn will be analysed as previously reported by Rasanen et $a l^{10}$ (monoclonal glyFn antibody) 
by DiabetOmics, Beaverton, Oregon, USA. The maternal glyFn and 'early' OGTT $75 \mathrm{~g}$ results are blinded to the investigators.

\section{Unblinding}

Values will be unblinded if the FPG levels are $\geq 7.0 \mathrm{mmol} / \mathrm{L}$ or the 2-hour plasma glucose levels are $\geq 11.1 \mathrm{mmol} / \mathrm{L}$, suggesting pre-existing diabetes mellitus. ${ }^{11}$ The diagnosis of pre-existing diabetes mellitus needs to be confirmed by an elevated glycosylated haemoglobin (HbA1c) value $\geq 6.5 \%$. Plasma glucose levels of $\leq 2.5 \mathrm{mmol} / \mathrm{L}$ are also abnormal and require further clarification. Women with confirmed pre-existing diabetes mellitus are treated according to a standardised protocol in line with current recommendations.

\section{Study outcomes}

Diagnosis of GDM

GDM is diagnosed if at least one value of the $75 \mathrm{~g}$ OGTT at 24-28 weeks of gestation exceeds the recommended IADPSG threshold: FPG of $\geq 5.1 \mathrm{mmol} / \mathrm{L}(92 \mathrm{mg} / \mathrm{dL})$, 1-hour glucose of $\geq 10.0 \mathrm{mmol} / \mathrm{L} \quad(180 \mathrm{mg} / \mathrm{dL})$ and 2-hour glucose of $\geq 8.5 \mathrm{mmol} / \mathrm{L} \quad(153 \mathrm{mg} / \mathrm{dL})$. All women who screen positive are followed up by a nutritionist and a diabetic nurse in contact with a diabetologist, and have frequent regular appointments in our obstetrical outpatient clinic in 2-4 weeks intervals depending on clinical condition, glucose values and ultrasound scan findings. Women who fail to meet the target glucose values after 1-2 weeks of diet management are treated with insulin according to the guidelines of the Swiss Society for Endocrinology and Diabetology (SGED), the Austrian Diabetes Association (ÖDG), the German Diabetes Association (DDG) and the German Association of Gynaecology and Obstetrics (DGGG). ${ }^{12-14}$ The glycaemic targets, insulin therapy, dose adjustments, concomitant medication and/or supplements are recorded.

\section{Pregnancy, delivery and neonatal outcome data}

Maternal data such as pre-eclampsia (blood pressure (BP) $\geq 140 / 90 \mathrm{~mm} \mathrm{Hg}>20$ weeks of gestation with proteinuria), pregnancy-induced hypertension ( $\mathrm{BP} \geq 140$ / $90 \mathrm{~mm} \mathrm{Hg}>20$ weeks of gestation, rate of sonographic estimated polyhydramnios (amniotic fluid index $\geq 25 \mathrm{~cm}$ ) or LGA (estimated birth weight $\geq 90$ th centile), delivery outcome including delivery mode (spontaneous vaginal, forceps, vacuum, planned caesarean section or during labour) and indication, and neonatal outcome data such as birth weight, rate of LGA (birth weight $\geq 90$ th centile), preterm birth $\leq 37$ completed weeks of gestation, 5 and $10 \mathrm{~min}$ Apgar scores, arterial umbilical cord $\mathrm{pH} \leq 7.0$, shoulder dystocia, birth trauma, neonatal hypoglycaemia (glucose value of $<2.5 \mathrm{mmol} / \mathrm{L}$ in infants born $\geq 34$ weeks of gestation), jaundice (transcutaneous bilirubin $>95$ th centile or need of phototherapy at any time after delivery), respiratory distress syndrome, congenital anomalies and admission to intensive care unit are prospectively collected.

\section{Statistics}

Sample size justification

We aim to demonstrate that the recently reported biomarker, glyFn and/or the 'early' OGTT $75 \mathrm{~g}$ at 12-15 weeks of gestation has sufficient diagnostic power to evaluate women at risk of developing GDM compared with the OGTT $75 \mathrm{~g}$ at 24-28 weeks of gestation. The receiver operator characteristic (ROC) curve of glyFn has a reported AUC of 0.91 (95\% CI 0.87 to 0.96$).{ }^{15}$ The OGTT $75 \mathrm{~g}$ screening test using the IADPSG criteria has not been tested in early pregnancy so far. The FPG value in early pregnancy has an AUC of 0.61 (95\% CI 0.54 to 0.68 ) compared with IADPSG criteria in later pregnancy in a retrospective study. ${ }^{16}$ We assumeaccording to data from the centre in Basel $^{17}$-that around $0.6 \%$ of recruited women will be diagnosed with pre-existing diabetes mellitus by the 'early' OGTT $75 \mathrm{~g}$. The prevalence of GDM is assumed to be around $10.9 \%$ according to a current IADPSG screening study from various Swiss laboratories. ${ }^{18}$ The new screening approach should have a proposed true AUC of 0.9 with a lower boundary of $0.8(95 \%$ CI $>0.8)$ which would lead to a power of $90 \%$ and an $\alpha$-level of $5 \%$ to an estimated sample size of 650 (65 women with GDM, 585 women without GDM). This power calculation is valid for OGTT, glyFn or combined markers. It ensures that the AUC is estimated with good precision regardless of the chosen biomarker or any combination. Offsetting a dropout of $15 \%$ leads to a total sample size of 748 . The dropout rate is expected to be equally distributed between centres. A sample size review will be performed after the first 300 recruitments. The power calculation was performed using MedCalc V.15.11 2015. ${ }^{19}$

\section{Statistical analysis plan}

Descriptive statistics and graphical examination will be performed for all primary and secondary study variables.

\section{Primary objective}

In order to predict GDM, ROC curves with corresponding AUCs will be calculated separately for glyFn and the 'early' OGTT $75 \mathrm{~g}$. GDM diagnosis by OGTT $75 \mathrm{~g}$ at 2428 weeks of gestation is considered a routine method. AUCs will be estimated with a $95 \%$ CI.

\section{Secondary objectives}

Logistic regression will allow combining glyFn, the 'early' OGTT and maternal risk factors in a multivariable risk model. Subsequent AUC will be calculated with $95 \%$ CI. Results will be internally cross-validated to prevent overoptimistic results. Optimal cut-off points to predict GDM will be determined based on these ROC curve. Sensitivity, specificity, positive and negative predictive values will also be estimated with $95 \%$ CI. However, other 'machine learning' algorithms could be better than 
logistic regression. Therefore, other popular procedures will be additionally tested: random forest and penalised logistic regression (Lasso). Details are described in Hastie et $a l^{20}$ Predictive performances will be internally crossvalidated and explored in examining AUC of the ROC and predicted versus observed probabilities. Random forest and penalised logistic regression avoid overfitting (to certain extent) whereas logistic regression does not. Random forest will be chosen because of its popularity and good benchmark results. Lasso is known for its good interpretability. Logistic regression will probably show its inferiority compared with the other methods. Internal cross-validation will be carried out using the package ' $\mathrm{mlr}$ ' within R. Internal cross-validation (eg, 10-fold) is a good possibility to estimate the fitting on a potential future data set. Based on these results, it will be decided whether a new prediction model for GDM will be proposed. In order to potentially improve the primary study variables, secondary study parameters such as changes in maternal body mass index (BMI) and/or clinical conditions like chronic hypertension and pregnancy-induced hypertension or pre-eclampsia and fetal conditions including intrauterine growth restriction will be added to the machine learning models. Statistical analyses will be performed and graphs will be plotted using the current version of the statistical computation program $\mathrm{R}$ ( $\mathrm{R}$ Development Core Team R. R: A Language and Environment for Statistical Computing. R Found Stat Comput 2014;1:409).

\section{Data recording}

Each participant receives an identification number to ensure confidentiality and the collected data are exchanged between centres using only the identification number. The name and birth date of each participant are stored with a different identification number in order to preserve the possibility to look for inconsistencies during the study.

\section{Reporting of adverse events}

Any (serious) adverse events (AE/SAE) are recorded by the investigator using the specific $\mathrm{AE} / \mathrm{SAE}$ sheet of the clinical report form. All SAE are reported to the responsible ethics committee within an appropriate time frame.

\section{OTHER STUDY MEASUREMENTS \\ Other biomarkers}

An extensively studied biomarker for early GDM screening is adiponectin. ${ }^{21-23}$ Adiponectin is an adipocytederived hormone and reflects whole body insulin sensitivity. ${ }^{24}$ A recently published meta-analysis calculated a summary sensitivity of $60.3 \%$ and a specificity of $81.3 \%$ with an AUC of $0.79 .{ }^{22}$ Maternal serum adiponectin concentration is measured by a quantitative sandwich ELISA technique. Another potential biomarker is pregnancyspecific glycoprotein-1 (PSG-1).$^{25}$ PSG-1 had a detection rate of $74 \%$ with a false-positive rate of $6 \%$ and an AUC of 0.81. PSG-1 is analysed as previously reported by Nagalla $e t a l^{25}$ by DiabetOmics, Beaverton, Oregon, USA.

\section{Evaluation of insulin and $\mathrm{HbA1c}$}

At the early study visit at 12-15 weeks of gestation, insulin, $\mathrm{C}$ peptide and $\mathrm{HbAlc}$ are additionally measured from the fasting blood sample in all women. HbAlc is measured by reversed-phase cation exchange chromatography (ADAMS HA-8160, Menarini Diagnostics Benelux, Zaventem, Belgium) or high performance liquid chromatography with Variant II, Bio-Rad (International Federation of Clinical Chemistry (IFCC) standardised and Diabetes Control and Complications Trial (DCCT) aligned with coefficient of variation (CV) $1.8 \%$ for HbAlc $=5.6 \%)$. Insulin is measured by chemiluminescence immunoassay (CLIA; Roche Modular E170, Basel, Switzerland). The homoeostasis model assessment of insulin resistance or the Quantitative Insulin Sensitivity Check Index (QUICKI) is used as an approximate of fasting (ie, hepatic) insulin resistance. ${ }^{26} 27$ The $\beta$-cell function is assessed from fasting glucose and insulin levels according to Wallace $e t a l^{26}$

\section{Measurement of vitamin D}

Vitamin D deficiency is associated with inhibited insulin secretion, insulin resistance and $\beta$-cell dysfunction in the pancreas in T2DM. ${ }^{28} 29$ Additionally, vitamin D has immunomodulatory properties which protects against the development of T1DM. Supplementation of $1,25-\mathrm{OH}$ vitamin $\mathrm{D}$ seems to alter $\mathrm{T}$ cells composition and reduces cytokine-induced apoptosis of pancreatic islet cells. ${ }^{30} 31$ We would like to have the actual values for $25-\mathrm{OH}$ vitamin $\mathrm{D}$ and it will be evaluated in this cohort by CLIA (Liaison, DiaSorin, Saluggia, Italy).

\section{Influence of stress in early pregnancy on development of GDM}

Psychological factors in early pregnancy might contribute to adverse obstetric outcome. ${ }^{32}$ This trial investigates the influence of perceived stress, stressful life events and depression on the development of GDM. The participants recruited in Basel are therefore asked to collect salivary samples for cortisol directly at the time of waking up, at 30 and at $60 \mathrm{~min}$ after waking up. The saliva samples are stored at $-20^{\circ} \mathrm{C}$ until analysis. After thawing, the saliva samples are centrifuged. Cortisol levels are determined using a competitive solid phase time-resolved fluorescence immunoassay with fluorometric end point detection (DELFIA). ${ }^{33}$ Copeptin is a more stable precursor hormone of arginine-vasopressin and is found to be elevated in many diseases. ${ }^{34-37}$ This effect can be attributed to the response of the hypothalamic-pituitary-adrenal axis to psychological stress. Copeptin will be measured in the fasting blood sample with time-resolved amplified cryptate emission technology (TRACE) by BRAHMS Kryptor Compact immunoanalyser from Thermo Scientific Brahms GmbH, Henningsdorf, Germany.

Three self-administered questionnaires are obtained at the study visit at $12-15$ weeks of gestation: 


\section{Questionnaire on perceived stress}

The perceived stress scale is a 10-item inventory for measuring the perception of stress. ${ }^{38}$ It is widely used and has been validated in pregnancy. ${ }^{39}$

\section{Questionnaire on stressful life events}

The Holmes and Rahe Stressful Life Events Scale $(\mathrm{SLE})^{40}$ is a 43-item instrument for the evaluation of stressful experiences during the past 12 months prior to answering the questionnaire.

\section{Questionnaire on depression}

The 10-item Edinburgh Postnatal Depression Screen $(\text { EPDS })^{41}$ is used to assess symptoms of depression during the past 7 days. It has been widely validated in pregnancy ${ }^{42} 43$ and is a part of the standard evaluation of pregnant women attending routine prenatal visits at the University Hospital in Basel. Scores $\geq 13$ indicate at least probable minor depression and scores $\geq 15$ indicated probable major depression. Pregnant women with scores $\geq 13$ are routinely offered psychological or psychiatric counselling during pregnancy.

\section{Histological examination of the placenta}

The fetal nutrients supply is regulated by maternal-fetal glucose and lipid concentration, placental blood flow and trophoblastic nutrient transporters. ${ }^{44}$ The placenta reacts with adaptive changes in structure and functions to a hyperglycaemic milieu. ${ }^{45}$ These changes of the placenta will be assessed depending on the maternal glycaemic control by standard pathology examination. ${ }^{46}$

\section{DISCUSSION}

To the best of our knowledge, this is the first large European cohort study that prospectively evaluates the promising new biomarker, glyFn, and the 'early' OGTT $75 \mathrm{~g}$ by comparing the impact of different GDM screening strategies with IADPSG criteria at 24-28 weeks of gestation. The IADPSG criteria have not been tested prospectively in early pregnancy, despite the suggestion of the IADPSG consensus panel in $2010^{8}$ to take a FPG value $\geq 5.1 \mathrm{mmol} / \mathrm{L}$ as a cut-off value for GDM. This recommendation was based on a retrospective study observing that high first trimester FPG in early pregnancy was associated with adverse pregnancy outcome. ${ }^{47}$ We propose that glyFn with FPG alone or in combination with postload glucose values should at least result in an AUC of 0.8 compared with the OGTT $75 \mathrm{~g}$ in later pregnancy. Rasanen et $a l^{15}$ could show that glyFn was independent of maternal age, parity, gestational age, the time of sample collection and the administration of the OGTT at 24-28 weeks of gestation. The current trial might clarify whether glyFn is dependent on maternal BMI or clinical conditions such as chronic hypertension, pregnancy-induced hypertension or the development of pre-eclampsia or intrauterine growth restriction.
The screening approach combining glyFn \pm FPG could overcome some problems of the OGTT. First, OGTT is time consuming. GlyFn and FPG alone can be drawn in a fasting state of the women in early morning and the women do not have to wait further. Second, OGTT is inconvenient to administer and some women suffer intolerance to the glucose load resulting in nausea and vomiting. No glucose administration would be necessary with a screening method combining glyFn and FPG. Third, we propose that glyFn might overcome the problem of low reproducibility like the OGTT. Like HbAlc, glyFn might also assess long-term serum glucose concentration. But this is hypothetical and needs to be proven. Additionally, we suppose that a multivariable prediction model incorporating risk factors, that is, maternal age and/or BMI, together with glyFn and/or FPG and postload glucose values might improve risk stratification in early pregnancy and could possibly decrease the required OGTTs later in pregnancy.

In the current study, the diagnostic power of glyFn will be evaluated using serum samples. But glyFn can be analysed additionally out of a dried blood stain. The resulting test is affordable (ie, estimated costs in India are US \$2-US $\$ 3 /$ dried blood spot, in Europe US\$20-US\$30/ serum sample), which would help especially developing countries that suffer particularly from problems with the implementation of the IADPSG recommendations, ${ }^{48}$ to benefit from a possibly simple and cheap screening tool for GDM. The analysis of glyFn in dried blood is not part of this trial and needs to be validated separately in future studies.

Cost-effectiveness analyses of IADPSG criteria using decision analysis models showed that the one-step screening with OGTT $75 \mathrm{~g}$ might be cost-effective when postdelivery care would reduce the development of T2DM in the mothers. ${ }^{49}{ }^{50}$ Another study reported costeffectiveness if pre-eclampsia would decrease to $>0.55 \%$ and caesarean delivery rate would fall to $>2.7 \% .{ }^{51}$ A new screening in the first trimester could be cost-effective if the method would reduce first the 1 and 2 hours blood sampling and/or second would decrease laboratory workload by avoiding a second screening in 24-28 weeks of gestation. Additionally, the newfound screening approach could result in the identification of women with overt diabetes or/and GDM in the first trimester. The aim of an early GDM diagnosis is the start of timely intervention with diet, exercise or-if necessary-insulin therapy in early second trimester. Earlier treatment potentially should result in the reduction of neonatal and maternal morbidities, that is, physical exercise reduces total maternal weight gain and the rate of GDM. ${ }^{52}$ However, there is still a paucity of randomised-controlled interventional trials showing that diagnosis and treatment of GDM $<24$ weeks of gestation improve pregnancy outcomes. Until the efficacy of early treatment is not studied and verified thoroughly, cost-effectiveness analysis will be of restricted value, but a cost analysis could be performed assuming different outcome scenarios. 
One limitation of our trial is that it is not powered for the evaluation of neonatal outcomes like LGA infants, neonatal hypoglycaemia, shoulder dystocia or birth trauma (these are planned secondary outcomes). The study would need to be significantly larger to detect effects on these neonatal outcomes. If this trial were to show positive diagnostic power for the new screening algorithm, a large multicentre study may be required to be sufficiently powered to determine the algorithm's effect on the rate of LGA infants or neonatal hypoglycaemia as proposed in the HAPO study. ${ }^{4}$

Finally, the glyFn assay is not yet commercially available and will be performed by DiabetOmics, Beaverton, Oregon, USA. For a widespread implementation of the finally proposed screening approach, a standardisation between laboratories will be necessary first.

The results of our study may have a major impact on future screening approaches for GDM by the development of a potentially simple, cost-effective and for the pregnant women a comfortable screening method for GDM in the first trimester.

\section{Author affiliations}

${ }^{1}$ Department of Obstetrics and Gynaecology, University Hospital Basel, Basel, Switzerland

${ }^{2}$ Department of Obstetrics and Gynaecology, Salzburger Landeskrankenhaus, Paracelsus Medical University, Salzburg, Austria

${ }^{3}$ Division of Obstetrics and Feto-maternal Medicine, Department of Obstetrics and Gynaecology, Medical University of Vienna, Vienna, Austria

${ }^{4}$ Department of Obstetrics and Gynaecology, Cantonal Hospital Aarau, Aarau, Switzerland

${ }^{5}$ Department of Obstetrics and Gynaecology, University Hospital Zurich, Zurich, Switzerland

${ }^{6}$ Department of Obstetrics and Gynaecology, University Hospital Freiburg, Freiburg, Germany

${ }^{7}$ Department of Pathology, University Hospital Basel, Basel, Switzerland

${ }^{8}$ Department of Biomedicine, Laboratory of Perinatology, University Basel,

Basel, Switzerland

Acknowledgements The authors would like to thank Diabet0mics, Beaverton, Oregon, USA for their support in the analysis of glyFn and the preparation of a previous version of this manuscript. The authors also would like to thank Dorothy Huang for critical proofreading in English and Cristina Granado and Doris Mueller Borer for their precious assistance in performing the study visits and in data acquisition. Special thanks to all families who participated in this study.

Contributors EAH is the coordinating investigator, study protocol author, obtained ethical approval and drafted this manuscript. IH is the sponsor, assisted with the original study protocol and revised the manuscript. SH and $\mathrm{EBr}$ designed the study together with $\mathrm{EAH}$ and revised the manuscript. AS performed the power calculation and statistical planning. TF, CSG, WE, PH, MKr, ED, NO-K, EBä, PH, MTB, MKu, RZ made important contributions and critically reviewed the content. All authors have given final approval of the version to be published.

Funding This study is supported by the Diabetes Society Basel, Switzerland and the Gottfried and Julia Bangerter-Rhyner-Foundation, Bern, Switzerland.

Competing interests None declared.

Ethics approval The study was approved by the local Institutional Review Boards in Basel (Ethikkommission Nordostschweiz (EKNZ)), Zurich, Freiburg, Salzburg and Vienna.

Provenance and peer review Not commissioned; externally peer reviewed.
Open Access This is an Open Access article distributed in accordance with the Creative Commons Attribution Non Commercial (CC BY-NC 4.0) license, which permits others to distribute, remix, adapt, build upon this work noncommercially, and license their derivative works on different terms, provided the original work is properly cited and the use is non-commercial. See: http:// creativecommons.org/licenses/by-nc/4.0/

\section{REFERENCES}

1. American Diabetes Association. (2) Classification and diagnosis of diabetes. Diabetes Care 2015;38 Suppl:S8-16.

2. Aziz NL, Abdelwahab S, Moussa M, et al. Maternal fructosamine and glycosylated haemoglobin in the prediction of gestational glucose intolerance. Clin Exp Obstet Gynecol 1992;19:235-41.

3. Crowther CA, Hiller JE, Moss JR, et al. Effect of treatment of gestational diabetes mellitus on pregnancy outcomes. $N$ Engl J Med 2005;352:2477-86.

4. Metzger BE, Lowe LP, Dyer AR, et al. Hyperglycemia and adverse pregnancy outcomes. N Engl J Med 2008;358:1991-2002.

5. Casey BM, Lucas MJ, Mcintire DD, et al. Pregnancy outcomes in women with gestational diabetes compared with the general obstetric population. Obstet Gynecol 1997;90:869-73.

6. Bellamy L, Casas JP, Hingorani AD, et al. Type 2 diabetes mellitus after gestational diabetes: a systematic review and meta-analysis. Lancet 2009;373:1773-9.

7. Osgood ND, Dyck RF, Grassmann WK. The inter- and intragenerational impact of gestational diabetes on the epidemic of type 2 diabetes. Am J Public Health 2011;101:173-9.

8. Metzger BE, Gabbe SG, Persson B, et al. International association of diabetes and pregnancy study groups recommendations on the diagnosis and classification of hyperglycemia in pregnancy. Diabetes Care 2010;33:676-82.

9. International Conference on Harmonisation of technical requirements for registration of pharmaceuticals for human use. ICH harmonized tripartite guideline: guideline for Good Clinical Practice. J Postgrad Med 2001;47(1):45-50.

10. Rasanen J, Quinn MJ, Laurie A, et al. Maternal serum glycosylated fibronectin as a point-of-care biomarker for assessment of preeclampsia. Am J Obstet Gynecol 2015;212:82.e1-9.

11. World Health Organization. Definition and Diagnosis of Diabetes Mellitus and Intermediate Hyperglycemia. WHO, 2006. http://www. who.int/diabetes/publications/diagnosis_diabetes2006/en/

12. Lehmann R, Troendle A, Brändle M. [New insights into diagnosis and management of gestational diabetes mellitus: recommendations of the Swiss Society for Endocrinology and Diabetes]. Ther Umsch 2009;66:695-706.

13. Kleinwechter $\mathrm{H}$, Schäfer-Graf U, Bührer $\mathrm{C}$, et al. Gestational diabetes mellitus (GDM) diagnosis, therapy and follow-up care: practice Guideline of the German Diabetes Association (DDG) and the German Association for Gynaecology and Obstetrics (DGGG). Exp Clin Endocrinol Diabetes 2014:122:395-405.

14. Kautzky-Willer A, Bancher-Todesca D, Pollak A, et al. [Gestational diabetes mellitus]. Wien Klin Wochenschr 2012;124(Suppl 2):58-65.

15. Rasanen JP, Snyder CK, Rao PV, et al. Glycosylated fibronectin as a first-trimester biomarker for prediction of gestational diabetes. Obstet Gynecol 2013;122:586-94.

16. Corrado F, D'Anna R, Cannata ML, et al. Correspondence between first-trimester fasting glycaemia, and oral glucose tolerance test in gestational diabetes diagnosis. Diabetes Metab 2012;38:458-61.

17. Huhn EA, Massaro N, Streckeisen S, et al. Fourfold increase in prevalence of gestational diabetes mellitus after adoption of the new International Association of Diabetes and Pregnancy Study Groups (IADPSG) criteria. J Perinat Med 2016. doi:10.1515/jpm-2016-0099 [Epub ahead of print $10 \mathrm{AUg} 2016$ ].

18. Ryser Rüetschi J, Jornayvaz FR, Rivest R, et al. Fasting glycaemia to simplify screening for gestational diabetes. BJOG 2016. http://dx. doi.org/10.1111/1471-0528.13857 [Epub ahead of print].

19. Hanley JA, McNeil BJ. The meaning and use of the area under a receiver operating characteristic (ROC) curve. Radiology 1982;143:29-36.

20. Hastie T, Tibshirani R, Friedman J. The elements of statistical learning: prediction, inference and data mining. Springer Verlag, 2009.

21. Nanda S, Savvidou M, Syngelaki A, et al. Prediction of gestational diabetes mellitus by maternal factors and biomarkers at 11 to 13 weeks. Prenat Diagn 2011;31:135-41.

22. Iliodromiti S, Sassarini J, Kelsey TW, et al. Accuracy of circulating adiponectin for predicting gestational diabetes: a systematic review and meta-analysis. Diabetologia 2016;59:692-9. 
23. Fasshauer M, Blüher M, Stumvoll M. Adipokines in gestational diabetes. Lancet Diabetes Endocrinol 2014;2:488-99.

24. Yadav A, Kataria MA, Saini V, et al. Role of leptin and adiponectin in insulin resistance. Clin Chim Acta 2013;417:80-4

25. Nagalla SR, Snyder CK, Michaels JE, et al. Maternal serum biomarkers for risk assessment in gestational diabetes. A potential universal screening test to predict GDM status. Indian J Endocrinol Metab 2015;19:155-9.

26. Wallace TM, Levy JC, Matthews DR. Use and abuse of HOMA modeling. Diabetes Care 2004;27:1487-95.

27. Katz A, Nambi SS, Mather K, et al. Quantitative insulin sensitivity check index: a simple, accurate method for assessing insulin sensitivity in humans. J Clin Endocrinol Metab 2000;85:2402-10.

28. Norman AW, Frankel JB, Heldt AM, et al. Vitamin D deficiency inhibits pancreatic secretion of insulin. Science 1980;209:823-5.

29. Maestro B, Molero S, Bajo S, et al. Transcriptional activation of the human insulin receptor gene by 1,25 -dihydroxyvitamin $\mathrm{D}(3)$. Cell Biochem Funct 2002;20:227-32.

30. Takiishi T, Ding L, Baeke F, et al. Dietary supplementation with high doses of regular vitamin D3 safely reduces diabetes incidence in NOD mice when given early and long term. Diabetes 2014;63:2026-36.

31. Riachy $\mathrm{R}$, Vandewalle B, Moerman E, et al. 1,25-dihydroxyvitamin D3 protects human pancreatic islets against cytokine-induced apoptosis via down-regulation of the Fas receptor. Apoptosis 2006;11: $151-9$.

32. Alder J, Fink N, Bitzer J, et al. Depression and anxiety during pregnancy: a risk factor for obstetric, fetal and neonatal outcome? A critical review of the literature. J Matern Fetal Neonatal Med 2007;20:189-209.

33. Dressendörfer RA, Kirschbaum C, Rohde W, et al. Synthesis of a cortisol-biotin conjugate and evaluation as a tracer in an immunoassay for salivary cortisol measurement. $J$ Steroid Biochem Mol Biol 1992;43:683-92.

34. Christ-Crain M, Fenske W. Copeptin in the diagnosis of vasopressin-dependent disorders of fluid homeostasis. Nat Rev Endocrinol 2016;12:168-76.

35. Reinstadler SJ, Klug G, Feistritzer HJ, et al. Copeptin testing in acute myocardial infarction: ready for routine use? Dis Markers 2015;2015:614145.

36. Zhang R, Liu J, Zhang Y, et al. Association between circulating copeptin level and mortality risk in patients with intracerebral hemorrhage: a systemic review and meta-analysis. Mol Neurobiol 2016. doi:10.1007/s12035-015-9626-z [Epub ahead of print]

37. Viasus D, Del Rio-Pertuz G, Simonetti AF, et al. Biomarkers for predicting short-term mortality in community-acquired pneumonia: a systematic review and meta-analysis. J Infect 2016;72:273-82.
38. Cohen S, Kamarck T, Mermelstein R. A global measure of perceived stress. J Health Soc Behav 1983;24:385-96.

39. Silveira ML, Whitcomb BW, Pekow P, et al. Perceived psychosocial stress and glucose intolerance among pregnant Hispanic women. Diabetes Metab 2014;40:466-75.

40. Holmes TH, Rahe RH. The social readjustment rating scale. $J$ Psychosom Res 1967:11:213-18.

41. Cox JL, Holden JM, Sagovsky R. Detection of postnatal depression. Development of the 10-item Edinburgh Postnatal Depression Scale. Br J Psychiatry 1987;150:782-6.

42. Byrn M, Penckofer S. The relationship between gestational diabetes and antenatal depression. J Obstet Gynecol Neonatal Nurs 2015;44:246-55.

43. Ertel KA, Silveira M, Pekow $P$, et al. Prenatal depressive symptoms and abnormalities of glucose tolerance during pregnancy among Hispanic women. Arch Womens Ment Health 2014;17:65-72.

44. Desoye G, Gauster M, Wadsack C. Placental transport in pregnancy pathologies. Am J Clin Nutr 2011;94(Suppl):1896S-902S.

45. Jarmuzek P, Wielgos M, Bomba-Opon D. Placental pathologic changes in gestational diabetes mellitus. Neuro Endocrinol Lett 2015;36:101-5.

46. Bernischke K, Burton GJ, Baergen RN. Pathology of the Human Placenta. Vol Sixth, Springer-Verlag Berlin Heidelberg, 2012.

47. Riskin-Mashiah S, Younes G, Damti A, et al. First-trimester fasting hyperglycemia and adverse pregnancy outcomes. Diabetes Care 2009;32:1639-43.

48. Nielsen KK, de Courten M, Kapur A. The urgent need for universally applicable simple screening procedures and diagnostic criteria for gestational diabetes mellitus-lessons from projects funded by the World Diabetes Foundation. Glob Health Action 2012;5. doi:10.3402/ gha.v5i0.17277

49. Werner EF, Pettker CM, Zuckerwise L, et al. Screening for gestational diabetes mellitus: are the criteria proposed by the international association of the diabetes and pregnancy study groups cost-effective? Diabetes Care 2012;35:529-35.

50. Marseille E, Lohse N, Jiwani A, et al. The cost-effectiveness of gestational diabetes screening including prevention of type 2 diabetes: application of a new model in India and Israel. J Matern Fetal Neonatal Med 2013:26:802-10.

51. Mission JF, Ohno MS, Cheng YW, et al. Gestational diabetes screening with the new IADPSG guidelines: a cost-effectiveness analysis. Am J Obstet Gynecol 2012;207:326.e1-9.

52. Sanabria-Martínez G, García-Hermoso A, Poyatos-León R, et al. Effectiveness of physical activity interventions on preventing gestational diabetes mellitus and excessive maternal weight gain: a meta-analysis. BJOG 2015;122:1167-74. 